\section{Ventilation-Perfusion Scanning: Stuck in a Rut, Perhaps, but the Road Ahead Is Not So Clear}

TO THE EDITOR: The recent editorial by Michael Graham extolled the virtues of ventilation-perfusion (V/Q) SPECT and its greater accuracy than either planar V/Q imaging or CT pulmonary angiography (CTPA) for detecting pulmonary emboli (PE) (1). I respect Dr. Graham's feeling on this matter but strongly disagree with his conclusions. This is a subject that I have dealt with for many years, and I have a very differing viewpoint from that offered by Dr. Graham.

The problem is that U.S. physicians practice defensive medicine worrying about lawyers looking over their shoulders. Any patient diagnosed with the smallest of PEs will be given anticoagulation treatment. I fully agree that SPECT is more accurate than planar imaging. However, in our country, that leads to overdiagnosis and overtreatment, with the potential for $8 \%-10 \%$ complications associated with blood thinners. We must, therefore, avoid diagnosing on CTPA or V/Q SPECT these small PEs that many authors have shown should not be treated (2-4). Dr. Lawrence Goodman, a chest radiologist from Milwaukee, points out that the only reasons to treat small PEs are coexistent deep venous thrombosis, inadequate cardiopulmonary reserve, and chronic PE (5). He even mentions that there is good evidence that healthy people often pass small clots from the legs to the lungs every day and that the lung capillary bed traps the emboli, protecting the systemic circulation. So-in the United States, PE is a problem that should be handled by outcomes rather than the accuracy medicine that Dr. Graham is advocating. Of course, V/Q SPECT is more accurate, but it works to the detriment of the patient for small PEs that are unnecessarily treated in the United States.

At New York's Montefiore Medical Center, we perform more than 3,500 planar V/Q studies a year. In addition, for the past $5 \mathrm{y}$ we have used a more understandable trinary interpretive algorithm in place of the older probability interpretations. Outcomes, based on 3-mo follow-ups, have shown comparable results for both interpretations, with a false-negative rate of $1 \%-1.5 \%$ (6). More recently, we had the opportunity to review follow-up outcomes data on more than 3,000 planar V/Q studies and 1,800 CTPA studies. The outcomes for both procedures are essentially the same, at $1 \%-$ $1.5 \%$. The well-known multiinstitutional prospective study of Anderson et al. in Canada showed greater sensitivity for CTPA ( $17.7 \%$ vs. $11.7 \%$ for planar V/Q) but comparable outcomes of approximately $1 \%$ (7). Another outcomes study for V/Q SPECT had a similar $1.5 \%$ false-negative rate $(8)$. These studies clearly indicate that the smaller clots picked up on CTPA or V/Q SPECT do not need to be treated. They justify the continued use of planar $\mathrm{V} / \mathrm{Q}$, particularly in the United States.

Most importantly, there is an ongoing multiinstitutional study (NCT01455818, clinicaltrials.gov) centered in Ottawa headed by Drs. Philip Wells and Gregoire Le Gal that is randomizing treatment versus nontreatment for small PEs. Early results suggest no difference. If these results continue, U.S. physicians may be con-

COPYRIGHT (c) 2014 by the Society of Nuclear Medicine and Molecular Imaging, Inc. vinced to trust their clinical judgment and not treat uncomplicated small PEs. When that day arrives, I would certainly consider using the more sensitive SPECT for selective V/Q studies.

\section{REFERENCES}

1. Graham MM. Ventilation-perfusion lung imaging: stuck in a rut? J Nucl Med. 2014;55:1395-1396.

2. Wiener RS, Schwartz LM, Woloshin W. When a test is too good: how CT pulmonary angiograms find pulmonary emboli that do not need to be found. BMJ. 2013;347:f3368.

3. Suh JM, Cronan JJ, Healey TT. Dots are not clots; the over-diagnosis and overtreatment of PE. Emerg Radiol. 2010;17:347-352.

4. Sheh SH, Bellin E, Freeman KD, Haramati LB. Pulmonary embolism diagnosis and mortality with pulmonary CT angiography versus ventilation-perfusion scintigraphy: evidence of overdiagnosis with CT? AJR. 2012;198:1340-1345.

5. Goodman LR. Small pulmonary emboli: what do we know? Radiology. 2005;234: 654-658.

6. Glaser JE, Chamarthy M, Haramati LB, Esses D, Freeman LM. Successful and safe implementation of a trinary interpretation and reporting strategy for V/Q lung scintigraphy. J Nucl Med. 2011;52:1508-1512.

7. Anderson DR, Kahn SR, Rodger MA, et al. Computed tomographic pulmonary angiography vs. ventilation-perfusion lung scanning in patients with suspected pulmonary embolism: a randomized controlled trial. JAMA. 2007;298:2743-2753.

8. Leblanc M, Leveillée F, Turcotte E. Prospective evaluation of the negative predictive value of V/Q SPECT using ${ }^{99 m}$ Tc-Technegas. Nucl Med Commun. 2007;28:667-672.

Leonard M. Freeman

Montefiore Medical Center

111 E. 210th St.

Bronx, New York 10022

E-mail: Ifreeman@montefiore.org

Published online Nov. 7, 2014.

DOI: $10.2967 /$ jnumed.114.148148

TO THE EDITOR: The recent editorial by Michael Graham states that the reason ventilation-perfusion (V/Q) SPECT has been slow to be adopted in the United States is due to confusion about the approach to interpretation of the studies; however, he states that the issue has been resolved-that is, follow the European procedure guidelines, eliminate probability categories, and interpret the studies as the presence or absence of pulmonary embolism (1). Some of the data supporting these statements deserve further discussion. However, this letter will focus on one particular aspect, the ventilation agent used for SPECT. To support the thesis that SPECT should be standard, the editorial specifically references one prospective and two retrospective publications supporting the use of SPECT $(2-4)$. The ventilation agents used in the first two were ${ }^{81 \mathrm{~m}} \mathrm{Kr}$ and ${ }^{99} \mathrm{~m}$ Tc-Technegas (Cyclomedica Ltd.). Both are excellent ventilation agents; however, neither is available in the United States. The third publication did not use ${ }^{99 \mathrm{~m}} \mathrm{Tc}$-diethylenetriaminepentaacetic acid (DTPA) as stated in the editorial but rather evaluated ${ }^{99 \mathrm{~m}}$ Tc-macroaggregated albumin SPECT without a ventilation scan.

${ }^{133} \mathrm{Xe}$ and ${ }^{99 \mathrm{~m}} \mathrm{Tc}$-DTPA are by far the most commonly used radiopharmaceuticals for ventilation studies in the United States. Most imaging clinics use ${ }^{99 \mathrm{~m}} \mathrm{Tc}$-DTPA because it produces good image quality, can obtain multiple views that match perfusion 
projections, and does not have the radiation safety issues of ${ }^{133} \mathrm{Xe}$. The downside is that in patients with chronic obstructive pulmonary disease, multiple central hot spots caused by turbulent airway flow are often seen, which can result in poor-quality and even nondiagnostic images. This becomes a particular problem with SPECT. ${ }^{133} \mathrm{Xe}$ is used at many institutions because the entire ventilator cycle can be viewed, hot spots are not a problem, and delayed washout is a sensitive indicator of obstructive lung disease, the most common alternative diagnosis to pulmonary embolism. Dr. Graham states that he uses ${ }^{99 \mathrm{~m}} \mathrm{Tc}$-sulfur colloid aerosol. ${ }^{99 \mathrm{~m}} \mathrm{Tc}$-sulfur colloid and ${ }^{99 \mathrm{~m}} \mathrm{Tc}-$ pyrophosphate aerosols are used at very few institutions, and published data supporting their clinical use are extremely limited.

Technegas has been available in Australia and Europe for years. Since 1986, there have been approximately 180 scientific publications about this radiopharmaceutical, with overwhelmingly positive sentiment and data on its safety and clinical efficacy. Anyone who has seen images of ${ }^{99 \mathrm{~m}} \mathrm{Tc}$-Technegas compared with ${ }^{99 \mathrm{~m} T c-D T P A}$ aerosol or ${ }^{133} \mathrm{Xe}$ readily appreciates the clear superiority of Technegas. The Australian manufacturer has been trying to obtain Food and Drug Administration (FDA) approval for Technegas in the United States for several years. However, the FDA has made this extremely difficult. Even though most imaging clinics in the United States use ${ }^{99 m}$ Tc-DTPA aerosol, the FDA will not allow a direct comparison between the two. The reason given is that the FDA never approved $99 \mathrm{~m}$ Tc-DTPA for ventilation studies. We presently use it on an off-label basis. Therefore, the FDA is requiring that Technegas be compared with ${ }^{133} \mathrm{Xe}$, even though ${ }^{133} \mathrm{Xe}$ is used in a minority of imaging centers. In addition, the FDA has required a large multicenter protocol that must include at least 375 subjects with a final diagnosis positive for pulmonary embolism and 375 that are negative for pulmonary embolism. The protocol is complex, time-consuming, and expensive. As a result, the sponsor is having difficulty finding institutions willing to participate and patient accrual has been poor. Many predict that this study will never be completed and that we will not be able to use Technegas in the United States in the foreseeable future. The FDA is hindering good patient care in the United States and disregarding the extensive experience in Australia and Europe. A simple direct image comparison of Technegas with ${ }^{133} \mathrm{Xe}$ or ${ }^{99 \mathrm{~m}} \mathrm{Tc}-\mathrm{DTPA}$ aerosol is all that should be needed. Its safety has already been demonstrated by the experience worldwide.

I agree with Dr. Graham that V/Q SPECT should become the standard-that is, if we had a ventilation agent that would routinely provide good diagnostic SPECT images. Institutions with generally healthy patients who do not have cardiopulmonary disease may get away with using ${ }^{99 \mathrm{~m}}$ Tc-DTPA aerosol with SPECT in most patients, but for institutions with many cardiopulmonary disease patients, particularly those with chronic obstructive pulmonary disease or asthma, ${ }^{99 \mathrm{~m}} \mathrm{Tc}$-DTPA aerosol SPECT can be quite problematic.

The FDA should reexamine its complex protocol comparing Technegas and ${ }^{133} \mathrm{Xe}$ with the sponsor and devise a protocol that would simply examine the value of Technegas ventilation images versus ${ }^{133} \mathrm{Xe}$ or ${ }^{99 \mathrm{~m}} \mathrm{Tc}$-DTPA aerosol images, using a protocol that can be accomplished with a limited number of patients in a reasonably short time. The extensive literature should be part of the approval process.

In summary, I agree that SPECT, particularly SPECT/CT, is the future of V/Q imaging. However, we unfortunately are not there yet, mainly because the FDA has hindered that progress, and this has adversely affected state-of-the-art optimal patient care.

\section{REFERENCES}

1. Graham MM. Ventilation-perfusion lung scanning: stuck in a rut? J Nucl Med. 2014;55:1395-1396.

2. Gutte H, Mortensen J, Jensen CV, et al. Detection of pulmonary embolism with combined ventilation-perfusion SPECT and low-dose CT: head-to-head comparison with multidetector CT angiography. J Nucl Med. 2009;50:1987-1992.

3. Quirce R, Ibanez-Bravo S, Jimenez-Bonilla J, et al. Contribution of V/Q SPECT to planar scintigraphy in the diagnosis of pulmonary embolism. Rev Esp Med Nucl Imagen Mol. 2014;33:153-158.

4. Lu Y, Lorenzoni A, Fox JJ, et al. Noncontrast perfusion single-photon emission CT/CT scanning. Chest. 2014;145:1079-1088.

\author{
Harvey A. Ziessman \\ Johns Hopkins University \\ 601 N. Caroline St., Ste. 3231 \\ Baltimore, MD 21278 \\ E-mail: hziessm1@jhmi.edu
}

Published online Nov. 7, 2014.

DOI: 10.2967/jnumed.114.149203

REPLY: I thank Drs. Freeman and Ziessman for their comments on my editorial regarding SPECT V/Q imaging (1).

I understand Dr. Freeman's point that problems can arise if a test is too sensitive. It seems to me that there are two ways to approach the problem of detecting and reporting small emboli that are clinically insignificant and do not require therapy. One is to not detect them and the other is to appropriately report them. The use of a lower-sensitivity approach, such as planar ventilation-perfusion (V/Q) imaging, certainly will avoid detection of small emboli. However, there are moderate-sized emboli, particularly in more medial lung, that cannot be visualized with planar V/Q and are clinically significant. Currently, we really do not know much about the prognosis or the need for treatment of small emboli, and the only way this issue can be studied is by using V/Q SPECT. This is a significant point raised in the European Association of Nuclear Medicine guidelines (2). Once the significance of smaller pulmonary emboli is better established, V/Q SPECT guidelines will need to be refined to determine which patients need treatment. Even if the high sensitivity of V/Q SPECT results in a small number of people being treated for trivial disease, because of its higher specificity V/Q SPECT is also likely to result in a decreased number of patients being overtreated whose lung scans are "nondiagnostic," that is, not normal or high-probability (3). It is likely at Montefiore that few lung scans are nondiagnostic, but in the rest of the country this is not an uncommon outcome.

Dr. Ziessman is concerned that ${ }^{99 \mathrm{~m}} \mathrm{Tc}-\mathrm{Technegas}$ (Cyclomedica Ltd.) is required to obtain high-quality SPECT ventilation images that are needed as part of V/Q SPECT imaging. I agree with him that Technegas is the best agent, but aerosol imaging with ${ }^{99 \mathrm{~m}} \mathrm{Tc}$-sulfur colloid generates remarkably highquality tomographic scans in most patients. The approach we use in Iowa results in a set of high-quality planar images as well as the tomographic images, so the interpreter can always fall back on evaluating the planar images. I agree that we need to try to convince the Food and Drug Administration to approve Technegas, but in the meantime we should move ahead with aerosol ventilation imaging and broadly adopt V/Q SPECT imaging. 